\title{
Levels of expression of CD19 and CD20 in chronic B cell leukaemias
} Lia Ginaldi, Massimo De Martinis, Estella Matutes, Nahla Farahat, Ricardo Morilla,
Daniel Catovsky
Academic Department of Haematology and Cytogenetics, The

Royal Marsden Hospital and Institute of Cancer Research, London SW3, UK E Matutes

N Farahat

R Morilla

D Catovsky

Department of Internal Medicine, L'Aquila University, Via S Sisto 22/B, 67100 L'Aquila, Italy

L Ginaldi

M De Martinis

Correspondence to: Professor Daniel Catovsky, Academic Department of Haematology and Cytogenetics, The Royal Marsden Hospital, Fulham Road, London SW3 6JJ, UK; email: d.catovsky@icr.ac.uk

Accepted for publication 4 February 1998

\begin{abstract}
Aims-To investigate whether the antigen levels of the B cell lineage markers CD19 and CD20 can distinguish between normal and neoplastic B cells or characterise distinct expression patterns among the chronic B cell leukaemias.

Methods-Peripheral blood cells from 70 patients with $B$ cell disorders and 17 healthy donors were analysed by quantitative flow cytometry. Direct immunofluorescence staining was performed with phycoerythrin conjugated CD19 and CD20 monoclonal antibodies. Standard microbeads with different capacities to bind mouse immunoglobulins were used to convert the mean fluorescence intensity (MFI) values into number of antigen molecules/cell, expressed as antibody binding capacity (ABC).

Results-CD19 and CD20 ABC values in leukaemic $B$ cells differed from those of normal blood B lymphocytes. The results identified distinct profiles of CD19 and CD20 expression in the various types of $B$ cell leukaemias. In all leukaemias studied except hairy cell leukaemia (HCL), CD19 expression was significantly lower than the mean (SD) value in normal $B$ cells (22 (7) $\times 10^{3}$ molecules/cell), as follows: chronic lymphocytic leukaemia (CLL), 13 (7) $\times 10^{3}$; B prolymphocytic leukaemia (B-PLL), 16 (9) $\times 10^{3}$; splenic lymphoma with villous lymphocytes (SLVL), 15 (11) $\times$ $10^{3}$; mantle cell lymphoma (MCL), $10(7) \times$ $10^{3}$. In HCL there was strong CD19 expression $\left(38(16) \times 10^{3}\right)$. In contrast, the level of expression of membrane CD20 was higher than the mean (SD) value in normal $B$ cells $\left(94\right.$ (16) $\times 10^{3}$ molecules/ cell) in MCL $\left(123(51) \times 10^{3}\right)$; B-PLL $(129$ $\left.(47) \times 10^{3}\right)$; SLVL $\left(167(72) \times 10^{3}\right)$; and HCL $\left(312(110) \times 10^{3}\right)$; while it was significantly lower $\left(65(11) \times 10^{3}\right)$ in CLL compared with normal $B$ cells and the other $B$ cell leukaemias.

Conclusions-Quantitative determination of CD19 and CD20 may provide useful diagnostic information for the study of $B$ lymphoproliferative disorders. (F Clin Pathol 1998;51:364-369)
\end{abstract}

Keywords: quantitative flow cytometry; B lymphocytes; $B$ cell leukaemias; CD19 and CD20 antigens

It is well established that immunophenotyping is a powerful tool in the classification of lymphoid disorders, ${ }^{1}$ by confirming a suspected diagnosis and demonstrating a charac- teristic phenotypic profile. However, there is sometimes an overlap of antigen expression in the various B cell disorders, such as CD5 positivity in mantle cell lymphoma (MCL), ${ }^{2}$ and chronic lymphocytic leukaemia (CLL). It is also known that some antigens are expressed at different densities in leukaemia, ${ }^{34}$ as compared with normal B cells. As a result, quantitative flow cytometry can be employed to distinguish between leukaemic and normal cells, ${ }^{5}$ and to some extent to differentiate between the various $\mathrm{B}$ cell disorders. We have previously shown that quantification by flow cytometric techniques can disclose differences in CD3 and CD7 expression between normal and leukaemic $\mathrm{T}$ cells and among the various $\mathrm{T}$ cell leukaemias. ${ }^{6}$

In the context of immature B lineage malignancies, quantitative flow cytometry has been used successfully to distinguish between leukaemic blasts and normal B cell precursors. ${ }^{78}$ Most previous studies on B lymphoproliferative disorders have focused on the presence or absence of surface antigens on the leukaemic cells, a feature designated "asynchronous phenotype." Few have considered antigen density as an important biological characteristic of the neoplastic cells, for example low membrane immunoglobulins and CD20 in CLL, ${ }^{9-12}$ or high CD5 and low CD19 density in CLL cells compared with CD5 positive normal B lymphocytes. ${ }^{13} 14$

We have applied a quantitative flow cytometry method to evaluate the expression of two B cell antigens, CD19 and CD20, in a variety of B cell malignancies in order (1) to compare the expression of these antigens in normal and leukaemic B lymphocytes; (2) to identify any abnormal patterns of antigen expression resulting from the malignant status; and (3) to investigate whether these changes may be useful for differential diagnosis.

\section{Methods}

Peripheral blood samples from 70 patients with a $\mathrm{B}$ cell disorder were obtained at diagnosis. The leukaemias included: CLL, 23 cases; B cell prolymphocytic leukaemia (B-PLL), 12 cases; hairy cell leukaemia (HCL), 13 cases; splenic lymphoma with villous lymphocytes (SLVL), 13 cases; and mantle cell lymphoma (MCL), nine cases. The diagnosis was based on clinical features, cell morphology, and immunophenotyping. ${ }^{15}$ All leukaemias expressed the B cell markers CD19 and CD20; CD5 was expressed in all CLL and MCL cases, whereas CD25 was consistently positive only in HCL. 
Table 1 Mean ABC (antibody binding capacity) values $\times 10^{3}$ in normal peripheral blood $B$ lymphocytes and $B$ lineage leukaemias

\begin{tabular}{lllllll}
\hline Antigen & Normal B cells & CLL & PLL & MCL & SLVL & HCL \\
\hline CD19 & $22(7)$ & $13(7)$ & $16(9)$ & $10(7)$ & $15(11)$ & $38(16)$ \\
(p value) $^{\star}$ & & $<0.001$ & $<0.05$ & $<0.001$ & $<0.05$ & $<0.001$ \\
CD20 & $94(16)$ & $65(11)$ & $129(47)$ & $123(51)$ & $167(72)$ & $312(110)$ \\
(p value) $^{\star}$ & & $<0.001$ & $<0.01$ & $<0.05$ & $<0.001$ & $<0.001$ \\
\hline
\end{tabular}

Values are mean (SD); ${ }^{\star}$ comparison with normal peripheral blood B lymphocytes. CLL, chronic lymphatic leukaemia; HCL, hairy cell leukaemia; MCL, mantle cell lymphoma; PLL, prolymphocytic leukaemia; SLVL, splenic lymphoma with villous lymphocytes.

Peripheral blood lymphocytes from 17 healthy donors were used as controls to establish reference ranges.

The mononuclear cells tested were derived from cryopreserved samples isolated from heparinised peripheral blood by 1.077 density gradient centrifugation with Lymphoprep (Nycomed, Oslo, Norway) from both patients and controls. Cell viability was assessed by trypan blue staining, which was more than $80 \%$ in all samples.

IMMUNOSTAINING, FLOW CYTOMETRY ANALYSIS, AND QUANTIFICATION

Direct immunofluorescence staining was performed with phycoerythrin conjugated CD19
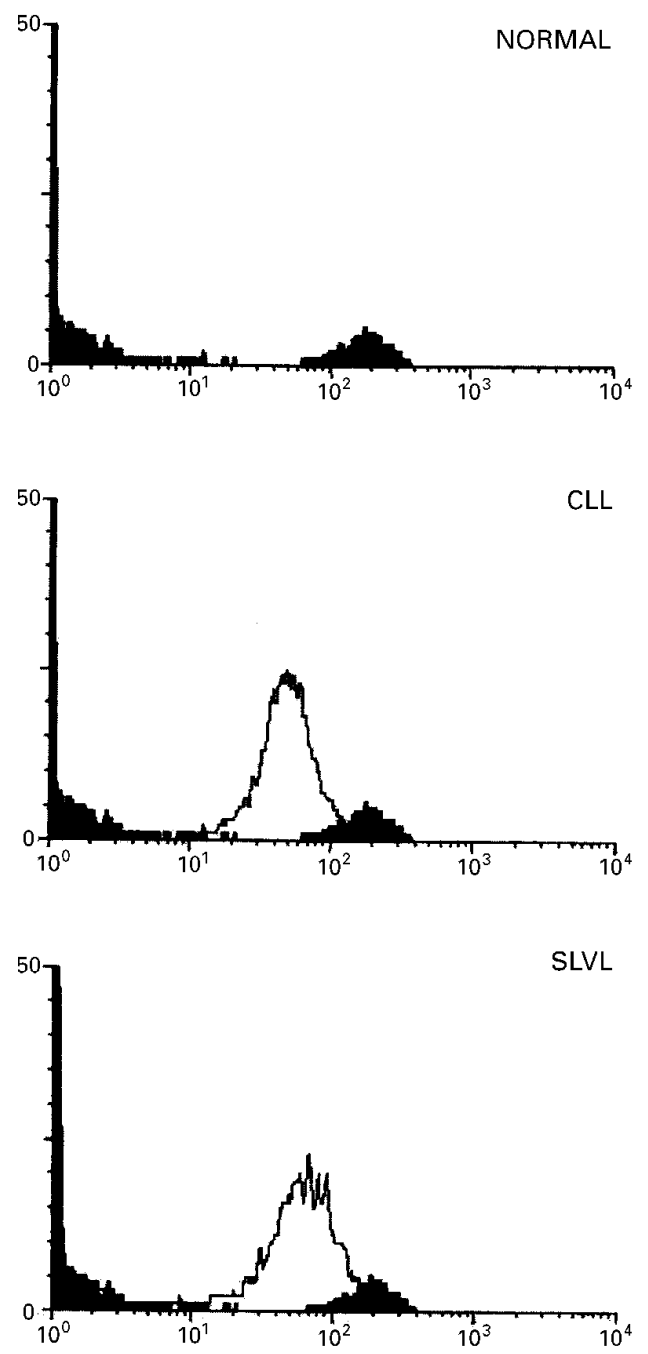

(B4-RD1) and CD20 (B1-RD1) monoclonal antibodies (Coulter Immunology, Hialeah, Florida, USA). The mononuclear cells were prepared for flow cytometry according to standard methods. ${ }^{5}$ Briefly, after washing three times with RPMI 1640 with $20 \%$ fetal calf serum (FCS), $1 \times 10^{6}$ cells were used per test. To each tube, $50 \mu 1$ of $2 \% \mathrm{AB}$ serum were added, followed by the saturating amount (10 $\mu \mathrm{l})$ of monoclonal antibodies. Appropriate directly conjugated isotypic antibodies (MsIgG-RD1; Coulter Immunology) were used as non-specific staining control in all experiments.

The cells were vortexed and incubated for 15 minutes at room temperature, washed three times with phosphate buffered solution (PBS)/ azide, and resuspended in $0.5 \mathrm{ml}$ Isoton (Coulter Immunology).

All studies were performed using a FACScan flow cytometer (Becton-Dickinson, Mountain View, California, USA) as previously described. ${ }^{16}$ The fluorescence intensity was measured with detectors and amplifiers set on a logarithmic scale. For each sample 10000 mononuclear cells were acquired for list mode
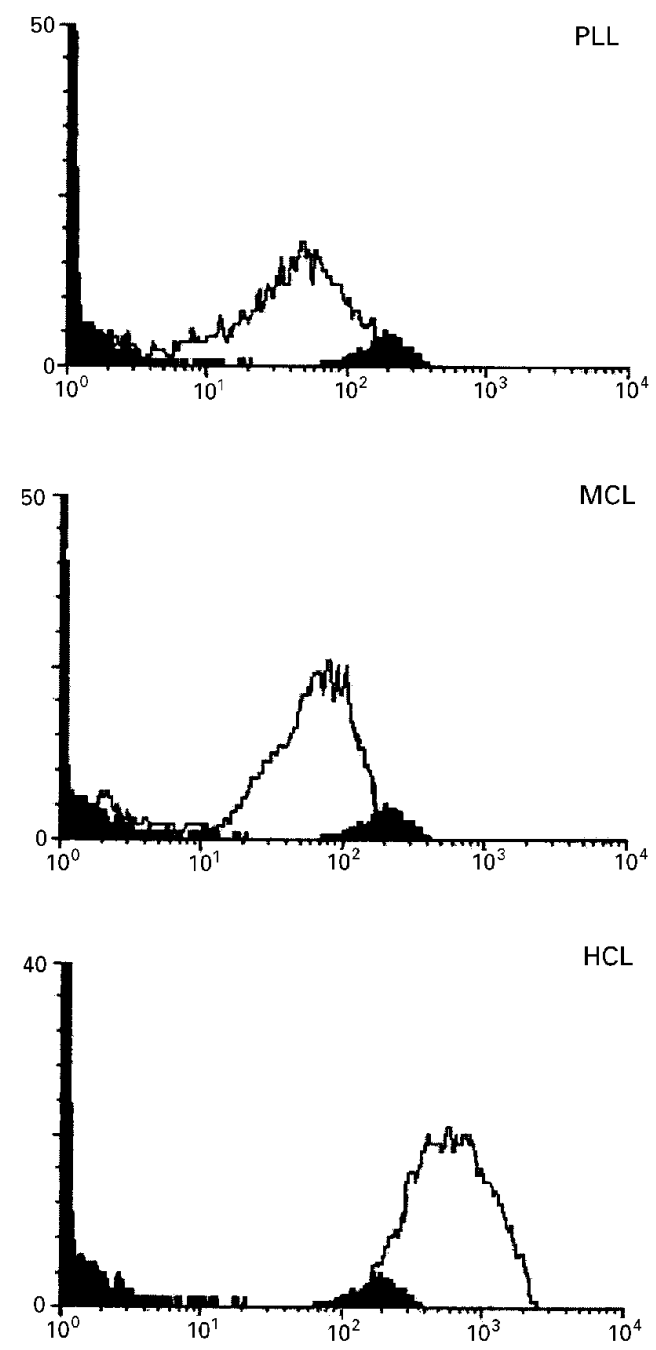

Figure 1 Overlays of single parameter histograms showing the difference in CD19 expression between normal peripheral blood B cells (displayed in black) and B cell leukaemias. CD19 is lower than in normal B lymphocytes in all B lineage leukaemias except HCL. 


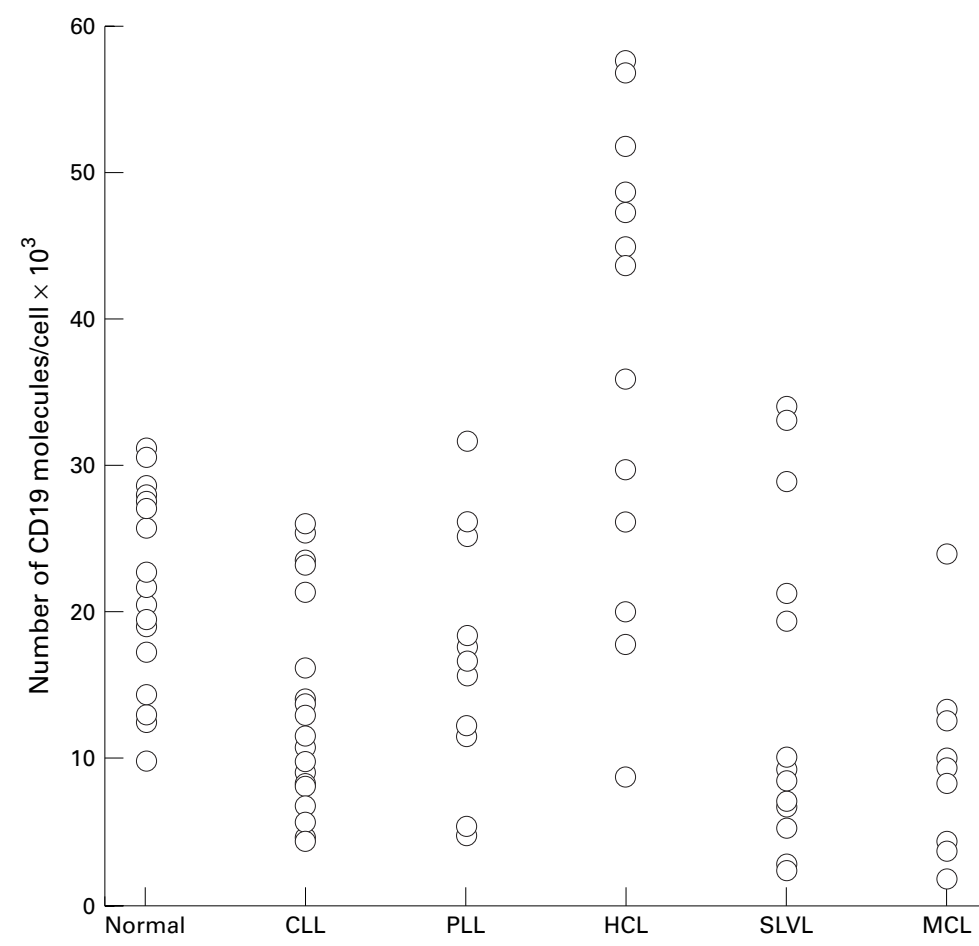

Figure 2 Distribution of individual $A B C$ values for $C D 19$ in normal peripheral blood B lymphocytes and $B$ cell leukaemias.

analysis by setting a live gate around the lymphocyte area in the forward scatter versus side scatter dot plot. Analysis was performed using Lysys II software (Becton Dickinson)

The mean fluorescence intensity (MFI) values of the positive cells were converted into number of molecules of antigen per cell or antibody binding capacity (ABC) by using the Quantum Simply Cellular (QSC) microbeads kit (Sigma, St Louis, Missouri, USA). This is a mixture of four microbead populations which differ by their incremental capacities to bind directly labelled mouse immunoglobulins. The accompanying software regresses the binding capacities of the microbeads against their corresponding peak channels. The regression curve permits quantitative estimates of the monoclonal antibody molecules bound to the target cells, that is, the number of molecules of antigen expressed per cell. ${ }^{17}$ Ten microlitres of each monoclonal antibody were added to $50 \mu \mathrm{l}$ QSC beads. After one hour of incubation, the mixtures were washed and analysed according to the method used for lymphocytes and using the same instrument's settings.

\section{STATISTICS}

The QUICKCAL software (FCSC; Sigma) was used for estimation of ABCs. The ABC values of CD19 and CD20 antigens in normal B lymphocytes and leukaemic cells were compared using Student's $t$ test for independent variables.

\section{Results}

The mean (SD) numbers of CD19 and CD20 molecules/cell in normal peripheral blood B lymphocytes were $22(7)$ and $94(16) \times 10^{3}$ respectively.
In cells from all the $\mathrm{B}$ lineage leukaemias except HCL, the mean number of CD19 molecules/cell was significantly lower than it was in normal B lymphocytes; in HCL, however, the CD19 ABC values were greater than in normal B cells or cells from the other B cell malignancies (table 1, figs 1 and 2).

The lowest CD19 density was found in MCL, followed by CLL, SLVL, and B-PLL, although no statistically significant differences were found between the mean values among these disorders. With a cut off point of $10 \times 10^{3}$ molecules/cell (the lowest normal value), five of nine MCL, seven of 13 SLVL, 10 of 23 CLL, and three of $12 \mathrm{~B}$-PLL samples had values $<10$, whereas only three cases had $\mathrm{ABC}$ values slightly over the normal range (fig 2). There were no differences between the 15 cases of typical CLL $\left(12(5) \times 10^{3}\right)$ and eight cases of CLL with atypical morphology $\left(16(8) \times 10^{3}\right)$. In contrast, a significantly larger mean number of CD19 molecules/cell was found in HCL than in normal B lymphocytes and the other B cell malignancies: eight of $13 \mathrm{HCL}$ cases had $\mathrm{ABC}$ values greater than $31 \times 10^{3}$ (the highest normal value) and none of them fell below the normal range (fig 2).

A significantly higher mean number of CD20 molecules/cell was found in all B cell malignancies compared with normal B lymphocytes, with the exception of CLL, in which the mean $A B C$ values was lower than in the other leukaemias and normal controls (table 1, figs 3 and 4). Only one CLL had a CD20 value within the normal range, whereas all other cases had CD20 ABCs below the normal range (fig 4). The number of CD20 molecules was lower in typical CLL $\left(15\right.$ cases; $\left.60(5) \times 10^{3}\right)$ than in CLL with atypical morphology (eight cases; $\left.74(13) \times 10^{3} ; \mathrm{p}<0.01\right)$. The levels in atypical CLL were still lower than in normal B cells and the other B cell leukaemias $(\mathrm{p}<0.05)$. B-PLL, SLVL, and MCL showed comparable CD20 antigen densities, although the values in SLVL were slightly higher (table 1, figs 3 and 4). The highest CD20 density was observed in HCL, in which the mean ABC value was significantly greater than in all other B cell leukaemias, with all HCL cases showing more than $31 \times 10^{3} \mathrm{CD} 20$ molecules/cell (the maximum normal value) (fig 4 ).

We have examined whether there was a correlation between the levels of CD19 and CD20 by the Spearman rank correlation test, but none was found. In addition, we have combined the results of CD19 and CD20 to see whether it has greater discriminatory power in distinguishing HCL from other B cell disorders, in particular SLVL, but no additional advantage was detected.

\section{Discussion}

CD19 and CD20 are widely expressed on normal and malignant B lymphocytes and thus they are not considered as discriminatory markers to distinguish among the different $\mathrm{B}$ cell malignancies. The development of techniques which allow the quantification of antigens by flow cytometry ${ }^{47}$ allowed us to quantify the levels of CD19 and CD20 

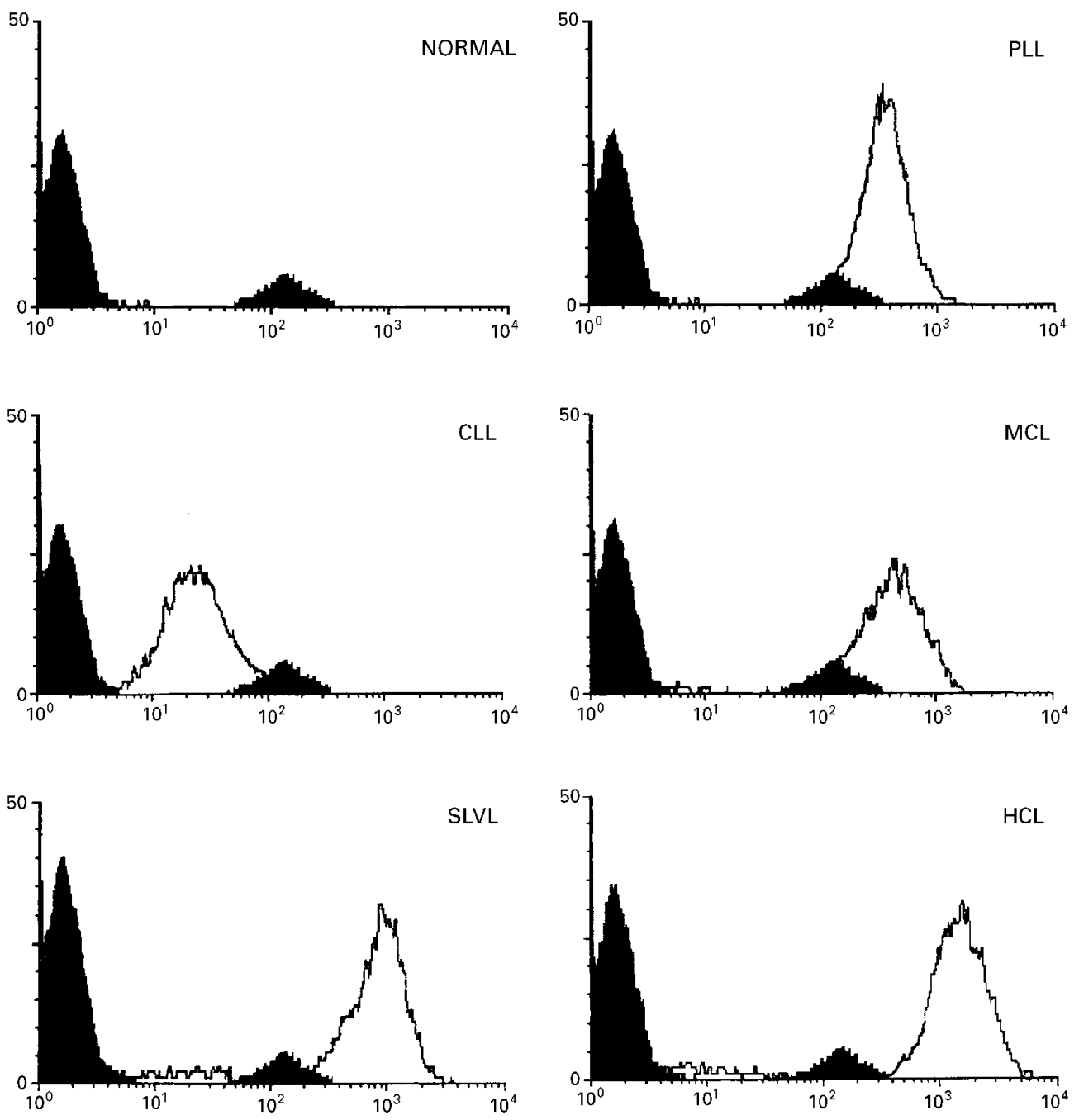

Figure 3 Overlays of single parameter histograms different CD20 expression patterns in B cell leukaemias compared with normal (full histogram). CLL has low CD20 density whereas in all other B cell leukaemias it is higher than normal. The highest $C D 20$ values are seen in $H C L$.

antigens in a range of chronic B leukaemias. Comparing both CD19 and CD20 density in normal and malignant $\mathrm{B}$ cell populations, it is possible to characterise these cells more precisely.

The two molecules investigated here have a functional role on B cells. CD19 serves as a coreceptor which modulates $\mathrm{B}$ cell growth and differentiation by the induction of cell cycle arrest or programmed cell death. ${ }^{18}$ The expression of CD19 on B cells is highly conserved and, together with CD79a, is a feature of early B cell progenitors. CD19 expression increases with differentiation through pre-B and mature $B$ cells, until it is downregulated at the later stages of B cell maturation, for example plasma cells. ${ }^{19}{ }^{20}$ The CD19 ABC values of peripheral blood B lymphocytes obtained in our study are comparable to those previously reported in bone marrow mature B cells. ${ }^{8}$

CD20 is a transmembrane phosphoprotein that functions as a calcium channel, ${ }^{21}$ and it has been shown to play an important role in B cell activation and differentiation. ${ }^{22}$ CD20 expression appears later than other B cell markers during normal B lymphocyte development and its membrane density progressively increases during differentiation. ${ }^{19} \mathrm{~B}$ cell precursors characteristically express a lower CD20 density than mature B cells. The lack or very low expression of CD20 is a known feature of B cell lineage ALL. ${ }^{5}$ In contrast, membrane CD20 increases either in vitro or in vivo during polyclonal B cell activation. ${ }^{22} 23$

We have shown here that cells from most chronic B cell malignancies, except HCL, show low CD19 expression. In contrast, a weaker CD20 expression compared with normal B cells was seen only on CLL lymphocytes, whereas strong CD20 was detected in the other $\mathrm{B}$ cell leukaemias, the highest value also being found in HCL. Therefore, our analysis has shown that, in combination with other antigenic markers, CD19 and CD20 might be useful to characterise B lymphoproliferative disorders with similar cytology. For example, the distinction between HCL and SLVL can sometimes be difficult based on the cytology of peripheral blood films, especially when the neoplastic cells make up a minor proportion of total leucocytes. Our findings suggest that these conditions can be distinguished by the 


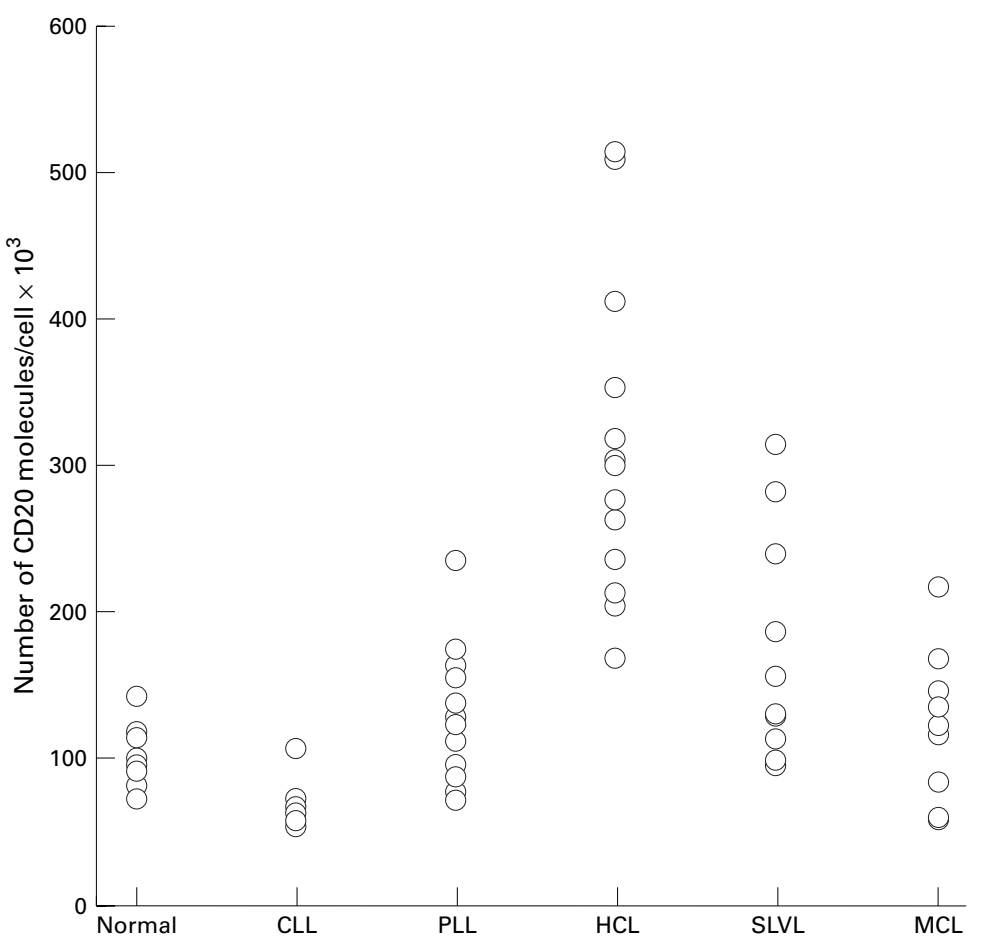

Figure 4 Individual $A B C$ values for $C D 20$ in normal peripheral blood $B$ cells and $B$ lineage leukaemias. cell development or represent abnormal phenotypes owing to neoplastic transformation.

This study shows that quantitative flow cytometry can detect differences in the levels of expression of the B cell markers CD19 and CD20 in B cell malignancies compared with normal B lymphocytes. Our results suggest that this approach may improve the discrimination between different disease entities and, in combination with morphology and other markers, enhance the accuracy in the diagnosis of B cell disorders.

NF was supported by a grant from the Egyptian Government. This work was funded in part by the Royal Marsden Hospital Trust Funds.

1 Matutes E, Owusu-Ankomah K, Morilla R, et al. The immunological profile of B-cell disorders and proposal of a scoring system for the diagnosis of CLL. Leukemia 1994;8:1640-5.

2 Pombo de Oliveira MS, Jaffe ES, Catovsky D. Leukaemic phase of mantle-zone (intermediate) lymphoma. Its characterisation in 11 cases. F Clin Pathol 1989;42: 962-72.

3 Peters RE, Janossy G, Ivory K, et al. Leukemia-associated changes identified by quantitative flow cytometry. III. B-cell gating in CD 37/k/1 clonality test. Leukemia 1994;8: B-cell gating

4 Bikoue A, George F, Poncelet P, et al. Quantitative analysis of leukocyte membrane antigen expression: normal adult values. Cytometry 1996;26:137-47.

5 Rothe G, Schmitz G, for the Working Group on Flow Cytometry and Image Analysis. Consensus protocol for the flow cytometric immunophenotyping of hematopoietic malignancies. Leukemia 1996;10:877-95.

6 Ginaldi L, Matutes E, Farahat N, et al. Differential expression of $\mathrm{CD} 3$ and $\mathrm{CD} 7$ in T-cell malignancies. A quantitative study by flow cytometry. Br f Haematol 1996; 93:921-7.

7 Lavabre-Bertrand T, Janossy G, Ivory K, et al. Leukemiaassociated changes identified by quantitative flow cytometry. I. CD10 expression. Cytometry 1994;18:209-17.

8 Farahat N, Lens D, Zomas A, et al. Quantitative flow cytometry can distinguish between normal and leukaemic cytometry can distinguish between normal and

9 Almasri NM, Duque RE, Iturraspe J, et al. Reduced expression of CD20 antigen as a characteristic marker for chronic lymphocytic leukemia. Am $\mathcal{F}$ Hematol 1992;40: 259-63.

10 Marti GE, Faguet G, Bertin P, et al. CD20 and CD5 expression in B-chronic lymphocytic leukemia. Ann N Y Acad Sci 1992;651:480-3.

11 Witzig TE, Li C-Y, Tefferi A, et al. Measurement of the intensity of cell surface antigen expression in B-cell chronic lymphocytic leukemia. Am f Clin Pathol 1994;101: 312-17.

12 Tefferi A, Bartholmai BJ, Witzig TE, et al. Heterogeneity and clinical relevance of the intensity of CD20 and immunoglobulin light-chain expression in B-cell chronic lymphocytic leukemia. Am f Clin Pathol 1996;106:457-61.

13 Lavabre-Bertrand T, Janossy G, Exbrayat C, et al. Leukemia-associated changes identified by quantitative flow cytometry. II. CD 5 over-expression and monitoring in B-CLL. Leukemia 1994;8:1557-63.

14 Cabezudo E, Matutes E, Ramrattan M, et al. Analysis of residual disease in chronic lymphocytic leukaemia by flow cytometry. Leukemia 1997;11:1909-14.

15 Bennet JM, Catovsky D, Daniel MT, et al. The FrenchAmerican-British (FAB) Cooperative Group. Proposals for the classification of chronic (mature) $\mathrm{B}$ and $\mathrm{T}$ lymphoid leukaemias. F Clin Pathol 1989;42:567-84.

16 Ginaldi L, Farahat N, Matutes E, et al. Differential expression of $\mathrm{T}$ cell antigens in normal peripheral blood lymphocytes: a quantitative analysis by flow cytometry. $\mathfrak{F}$ Clin Pathol 1996;49:539-44.

17 Lenkei R, Andersson B. Determination of the antibody binding capacity of lymphocyte membrane antigens by flow binding capacity of lymphocyte membrane antigens by flow cytometry in 58

18 Sato S, Steeber DA, Tedder TF. CD19 signal transduction molecule is a response regulator of B-lymphocyte differentiation. Proc Natl Acad Sci 1995;92:11558-62.

19 Loken MR, Shah VO, Dattilio KL, et al. Flow cytometric analysis of human bone marrow. II. Normal B-lymphocyte development. Blood 1987;70:1316-24.

20 Scheurmann RH, Racila E. CD19 antigen in leukemia and lymphoma diagnosis and immunotherapy. Leuk Lymphoma 1995;18:385-97.

21 Kanzaki M, Shibata H, Mogami $\mathrm{H}$, et al. Expression of calcium-permeable cation channel CD20 accelerates progression through the G1 phase in Balb/c 3T3 cells. F Biol gression through the G1 phase
Chem 1995;270:13099-104.

22 Tedder TF, Boyd AW, Freedman AS, et al. The B cell surface molecule B1 is functionally linked with B cell activation and differentiation. F Immunol 1985;135:973-9. sented in normal tissues. However, it is not certain whether the CD19 and CD20 ABC values in leukaemia reflect different stages of $\mathrm{B}$ 
23 Valentine MA, Keier KE, Rossie S, et al. Phosphorylation of the CD20 phosphoprotein in resting B lymphorytes: reguthe CD20 phosphoprotein in resting B lymphocyte

24 Matutes E, Morilla R, Owusu-Ankomah K, et al. The immunophenotype of hairy cell leukemia (HCL). Proposal for a scoring system to distinguish from B-cell disorders with hairy or villous lymphocytes. Leuk Lymphoma 1994;14:57-61.
25 Robbins BA, Ellison DJ, Spinosa JC, et al. Diagnostic application of two-color flow cytometry in 161 cases of hairy cell cation of two-color flow cytometry in

26 Visser L, Poppema S. Induction of B-cell chronic lymphocytic leukaemia and hairy cell leukaemia like phenotypes by phorbol ester treatment of normal peripheral blood B-cells. Br F Haematol 1990;75:359-65.

\title{
The Royal College of Pathologists, London SW1 Symposia in 1998
}

\author{
Antiviral Resistance-Clinical and Laboratory Update \\ Wednesday 13 May 1998
}

Calman Cancer Units-Minimum Standards for Dissecting and Reporting Thursday 21 May 1998

Malaria-Some Current Perspectives

Wednesday 3 fune 1998

Histopathology-Cytopathology External Quality Assessment: The Way Forward? Wednesday 10 June 1998

HLA in Transplantation and Disease

Thursday 2 fuly 1998

These meetings will be held at the Royal College of Pathologists, 2 Carlton House Terrace, London SW1Y 5AF, and are open to members of the College and non-members. Further details from the Scientific Meetings Officer, tel 0171930 5862, ext 24/25. 